\title{
Left ventricular twisting mechanics and exercise in healthy individuals: a systematic review
}

\author{
This article was published in the following Dove Press journal: \\ Open Access Journal of Sports Medicine \\ 17 August 2012 \\ Number of times this article has been viewed
}

\author{
C Taylor Drury ${ }^{1,2}$ \\ Shannon SD Bredin ${ }^{3}$ \\ Aaron A Phillips ${ }^{1,2}$ \\ Darren ER Warburton ${ }^{1,2}$ \\ 'Cardiovascular Physiology and \\ Rehabilitation Laboratory, University \\ of British Columbia; ${ }^{2}$ Experimental \\ Medicine Program, Faculty of \\ Medicine, University of British \\ Columbia; ${ }^{3}$ Cognitive and Functional \\ Learning Laboratory, University \\ of British Columbia, Vancouver, \\ BC, Canada
}

\begin{abstract}
The aim of this study was to review systematically the effects of exercise on left ventricular (LV) twisting mechanics in healthy individuals. Literature searches were conducted in electronic databases for articles reporting measures of LV twisting mechanics in healthy individuals before and during/after exercise. Upon review, 18 articles were analyzed. Studies were separated by exercise type into the following four categories to allow for detailed comparisons: submaximal, prolonged endurance, maximal, and chronic endurance. Despite an overall methodological quality of low to moderate and within-group variations in exercise intensity, duration, and subject characteristics, important trends in the literature emerged. Most important, the coupling of LV systolic twisting and diastolic untwisting was present in all exercise types, as both were either improved or impaired concomitantly, highlighting the linkage between systole and diastole provided through LV twist. In addition, trends regarding the effects of age, training status, and cardiac loading also became apparent within different exercise types. Furthermore, a potential dose-response relationship between exercise duration and the degree of impairment to LV twisting mechanics was found. Although some disagreement existed in results, the observed trends provide important directions for future research. Future investigations should be of higher methodological quality and should include consistent exercise protocols and subject populations in order to minimize the variability between investigations.
\end{abstract}

Keywords: twisting mechanics, diastolic untwisting, rotational parameters, exercise intensity and duration

\section{Introduction}

The left ventricle (LV) has three planes of motion: longitudinal, radial, and circumferential. ${ }^{1}$ Circumferential motion of the LV is the result of the contraction and relaxation of obliquely oriented epicardial and endocardial myofibres. ${ }^{2}$ These oblique contractions in the healthy human heart, when viewed from the apex, result in circumferential clockwise rotation at the base, and counterclockwise rotation at the apex. The net result of these opposing rotations in the basal and apical planes is a wringing motion of the heart during systole, referred to as LV twist. ${ }^{3}$ A rapid untwisting motion in the opposite direction occurs during diastole, ${ }^{4}$ and is believed to be caused by the release of elastic potential energy accumulated during the systolic contractions ${ }^{5}$ - likely from the compression of spring-like cardiac proteins such as titin. ${ }^{6}$ This early diastolic untwisting enhances the generation of the intraventricular pressure gradient and works to draw blood from the left atrium into the left ventricle, a process known as diastolic suction, and thereby enhances diastolic filling and allows diastolic filling to occur at lower atrial pressures. ${ }^{7}$ Taken together, LV twist, the corresponding diastolic
Correspondence: Darren ER Warburton Room 204, Unit II, Osborne Centre, Cardiovascular Physiology and Rehabilitation Laboratory, Physical Activity Promotion and Chronic Disease Prevention Unit, University of British Columbia, 6108 Thunderbird Boulevard, Vancouver, BC V6T IZ3, Canada

$\mathrm{Tel}+$ I 604822 I337

Fax +I 6048229222

Email darren.warburton@ubc.ca
(C) 2012 Drury et al, publisher and licensee Dove Medical Press Ltd. This is an Open Access article which permits unrestricted noncommercial use, provided the original work is properly cited. 
untwisting, and the apical and basal components of each can be viewed as the twisting mechanics of the left ventricle and serve as important indicators of LV function.

During exercise, LV twisting mechanics play an important role in augmenting cardiac function. It is well understood that the increases in chronotropic stimulation that occur with exercise result in reduced diastolic filling time, requiring a compensatory increase in diastolic filling in order to maintain cardiac output. As inotropy also increases during exercise, more potential energy is produced during systole through vigorous twisting contractions, resulting in greater diastolic untwisting and therefore allowing for diastolic filling to occur more rapidly. ${ }^{8}$ As diastolic untwisting plays a major role in diastolic filling, and relies heavily on LV twist, it is clear that the twisting mechanics of the left ventricle are integral to cardiac function during exercise. The measurement of these twisting mechanics under exercise conditions is therefore critical to an accurate understanding of the relationship between exercise and heart function.

Recent improvements in echocardiography and cardiac magnetic resonance imaging have allowed the twisting mechanics of the left ventricle to be measured and quantified in healthy individuals. ${ }^{9} 10$ Correspondingly, there has been a rapid increase in the number of papers examining the changes in LV twist and diastolic untwisting resulting from exercise. In light of this, the present authors feel that a systemic assessment of the overall findings from these articles is required. Two recent narrative reviews have discussed changes in various indicators of $\mathrm{LV}$ function that occur following acute and prolonged bouts of exercise. ${ }^{11,12}$ However, to the present authors' knowledge, no systematic review exists examining the effects of all forms of exercise on LV twisting mechanics. Accordingly, the primary purpose of this review is to systematically summarize and evaluate the existing literature examining the effects of exercise on LV systolic and diastolic twisting mechanics in healthy individuals. The aim is to provide clarity to the effects of different exercise durations and intensities on LV twisting mechanics, and the present authors hypothesize that acute short-term exercise will induce an increase in these LV parameters, while prolonged exercise will cause a transient decrease.

\section{Methods}

\section{Search strategy}

An extensive literature search on the effects of exercise on LV systolic and diastolic twisting mechanics was conducted in the following electronic databases: An extensive literature search on the effects of exercise on LV systolic and diastolic twisting mechanics was conducted in the following electronic databases (from 1950 - January 2012): MEDLINE, EMBASE, and EBSCO (Cochrane Library, ACP Journal Club, DARE, CCTR, CMR, HTA, NHSEED, Academic Search Complete, CINAHL, PsycINFO, and SPORTDiscus). The selected items were published after 1950 and before January 2012. Search terms were divided into three categories: (1) exercise, (2) left ventricle, and (3) twisting mechanics. The exercise search terms used were developed previously in systematic reviews conducted by the authors' research group. ${ }^{13}$ See Table 1 for a complete list of the Medical Subject Headings and keywords used.

\section{Screening}

A total of 127 articles were found through the literature search. Duplicates, review articles, conference abstracts, articles not in English, letters to the editor, studies investigating only pathological conditions (without data on healthy individuals), and investigations that did not report data on LV twisting mechanics were excluded, leaving 14 articles remaining. In addition, four articles were found via manual cross-referencing and the authors' knowledge of the subject area, resulting in a total of 18 articles for final review (Figure 1).

Table I Results of the MEDLINE literature search using the Ovid interface

\begin{tabular}{|c|c|c|c|}
\hline $\begin{array}{l}\text { Search } \\
\text { no }\end{array}$ & Searches (March I3, 20II)* & Results & \\
\hline 1 & exp Physical Fitness/ & 18,174 & Exercise \\
\hline 2 & exp Motor Activity/ & 93,010 & parameters \\
\hline 3 & exp Physical Endurance/ & 19,700 & \\
\hline 4 & exp Exercise/ & 54,165 & \\
\hline 5 & exp Physical Exertion/ & $50,|7|$ & \\
\hline 6 & exp Sports/ & 89,073 & \\
\hline 7 & exp Exercise Therapy/ & 22,408 & \\
\hline 8 & exp Exercise Tolerance/ & 6138 & \\
\hline 9 & exp Health Behavior/ & 75,536 & \\
\hline 10 & exp Heart Ventricles/ & 59,094 & LV \\
\hline II & exp Ventricular Function, Left/ & 22,147 & parameters \\
\hline 12 & exp Ventricular Function/ & 39,642 & \\
\hline 13 & Myocardial.mp. & 299,096 & \\
\hline 14 & Rotation\$.mp. & 68,499 & LV twisting \\
\hline 15 & Recoil\$.mp. & 2248 & parameters \\
\hline 16 & Twist\$.mp. & 9749 & \\
\hline 17 & Untwist\$.mp. & 404 & \\
\hline 18 & Torsion\$.mp. & 16,973 & \\
\hline 19 & $\begin{array}{l}\text { I or } 2 \text { or } 3 \text { or } 4 \text { or } 5 \text { or } 6 \text { or } \\
7 \text { or } 8 \text { or } 9\end{array}$ & 329,205 & \\
\hline 20 & 10 or 11 or 12 or 13 & 353,080 & \\
\hline 21 & 14 or 15 or 16 or 17 or 18 & 93,704 & \\
\hline 22 & 19 or 20 or 21 & 43 & \\
\hline
\end{tabular}

Notes: *Use of "l" after an index term indicates that all subheadings were selected; "\$" at the end of a term indicates that this term has been truncated; "exp" before an index term indicates that the term was exploded; ".mp." indicates a free text search for a term.

Abbreviation: LV, left ventricular. 


\section{Quality assessment and level of evidence}

Two reviewers (TD and AP) assessed all articles for methodological quality and level of evidence in duplicate, and any disagreements were settled through consultation with a third reviewer (DW) until 100\% consensus was achieved. All systematic review-related processes were overseen and directed by a senior investigator specializing in systematic reviews (SB). Methodological quality was determined using the Downs and Black ${ }^{14}$ Quality Index checklist, as the review did not include any randomized controlled trials. The Quality Index checklist was modified according to Prince et $\mathrm{a}^{15}$ (and used previously by the authors' research group ${ }^{13}$, to incorporate only the most relevant questions from the original checklist. Each article was assigned a score out of 15 , with higher scores indicating greater levels of methodological quality. Level of evidence was assessed using a five-point scale ${ }^{16}$ (modified from Sackett et al ${ }^{17}$ ). In brief, Level 1 (the highest level of evidence) corresponds to a randomized controlled trial of high quality. Level 2 refers to a randomized controlled trial of lower quality, a prospective controlled trial, or a cohort study. Level 3 is a case control study design. Level 4 refers to a pre-post, posttest, or case series study design. Level 5 (the lowest level of evidence) can be of observational, clinical consensus, or case report design.

\section{Results}

The 18 included articles involved a total of 324 healthy participants (276 men, 48 women) with an age range of 19-70 years. The articles were categorized by exercise type into four groups: (1) acute submaximal exercise (nine studies, 147 total participants); ${ }^{8,18-25}$ (2) prolonged endurance exercise (five studies, 102 total participants); ${ }^{26-30}$ (3) acute maximal exercise (three studies, 60 total participants); ${ }^{31-33}$ and (3) chronic endurance exercise (one study, 15 total participants). ${ }^{34}$ See Table 2 for an overall summary and

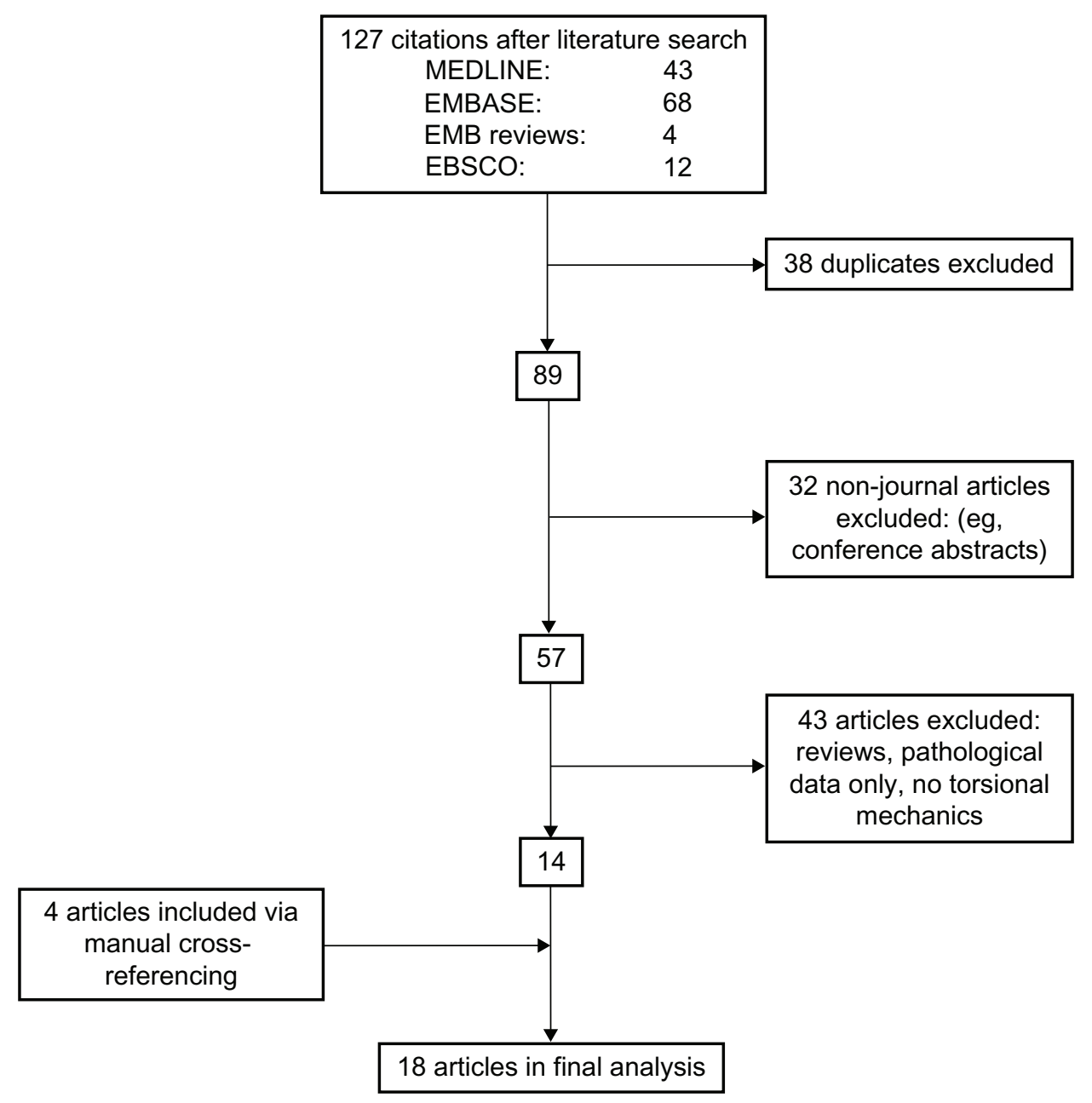

Figure I Flowchart of literature search process. Abbreviation: EMB, evidence-based medicine. 
Supplementary Tables 1-4 for a comprehensive summary of the articles in each exercise category. The articles examined were of low to moderate methodological quality, with a mean Downs and Black ${ }^{14}$ score of $10.61 \pm 0.85$ out of 15 (range, 9-12). The articles were published over a 9-year period (2003-2012).

\section{Acute submaximal exercise}

Nine studies investigated the effects of acute submaximal exercise on systolic and diastolic LV twisting mechanics (147 total participants; approximate mean ages: $20-70$ years). All investigations measured LV function at rest and during exercise. Seven investigations utilized either supine or semisupine bicycling as their exercise mode, ${ }^{8,18-21,24,25}$ while the two remaining investigations utilized a unilateral knee extensor exercise protocol. ${ }^{22,23}$

\section{Systolic parameters}

Acute submaximal exercise was found to result primarily in augmented LV systolic twisting mechanics (including LV twist, twisting rate, systolic apical and basal rotation, and apical and basal rotation rate) in individuals below the age of 40..$^{8,18-20,24-25}$ However, two investigations reported no change in any of these parameters in response to sub-maximal exercise in individuals of this age group. ${ }^{22,23}$ This disagreement is most likely due to the use of a weak exercise stimulus in these two outlying investigations, as unilateral knee extensor exercises did not induce increases in either stroke volume or ejection fraction, ${ }^{22,23}$ two indices of cardiac function that were found to increase in all other investigations in this category. Interestingly, one investigation examined the changes in LV twisting mechanics during submaximal exercise between endurance-trained and normally active individuals. ${ }^{25}$ This investigation reported that all measured systolic LV twisting mechanics increased from rest to exercise in both groups, but found no evidence of group differences in any LV twisting mechanic parameter, aside from lower apical rotation, and the endurance-trained group had a slightly lower LV twist $(P=0.09)$ at rest and during exercise than the normally active group. ${ }^{25}$ In addition, submaximal exercise was also found to primarily result in no change in LV systolic twisting mechanics in individuals above the age of $40,{ }^{18,20}$ although one investigation did report increased peak apical rotation in an older population during semi-recumbent cycling. ${ }^{21}$ This discrepancy is potentially attributable to differences in the selected sample populations, as the older populations examined by Burns et $\mathrm{al}^{18}$ and Esch et $\mathrm{al}^{20}$ were on various cardiac and blood pressure-lowering medications, while the population examined by Tan et $\mathrm{al}^{21}$ was free of any cardiovascular medications. The absence of medication possibly reflects a healthier cardiovascular system in which LV function may more closely resemble that of younger individuals in the ability to augment systolic twisting mechanics. In support of this theory, the peak heart rates elicited by the exercise protocols used by Burns et $\mathrm{al}^{18}$ and Esch et $\mathrm{al}^{20}$ were greater than that reported by Tan et $\mathrm{al}^{21}$ (140 and $110 \mathrm{bpm}$, respectively, versus $90 \mathrm{bpm}$ ). Observing an increase in peak apical rotation despite a lower peak exercising heart rate ${ }^{21}$ suggests that a greater intrinsic LV function, such as that found in younger individuals, was present in the sample population.

\section{Diastolic parameters}

Consistent with the increase in LV systolic twisting mechanics, diastolic twisting mechanics (including untwisting rate, time to peak untwisting rate, and diastolic apical and basal rotation rates) were generally reported to increase during submaximal exercise in individuals below the age of $40 .^{8,18-}$ 20,24-25 The same two investigations which utilized a lower exercise intensity reported no change in diastolic twisting mechanics. ${ }^{22,23}$ In endurance-trained and normally active individuals, submaximal exercise induced an increase in all measured LV diastolic twisting mechanics, yet there was no difference in any diastolic twisting mechanic parameter between groups at rest or during exercise. ${ }^{25}$ In individuals above the age of 40, submaximal exercise resulted primarily in impaired diastolic untwisting (increased time to peak untwisting rate, failure to augment peak untwisting rate). ${ }^{18,20}$ However, the same investigation that reported contradictory improvements in systolic twisting mechanics in a potentially healthier older population also found conflicting evidence of increased peak untwisting rate in these individuals. ${ }^{21}$ The percentage of LV untwisting that occurred prior to mitral valve opening, a marker of early diastolic function and an essential aspect of diastolic suction, was found to be primarily unchanged in individuals below the age of $40 .^{8,19,20}$ The two studies to report this measure in those older than 40 years found conflicting evidence, as Esch et $\mathrm{al}^{20}$ reported a decrease - further supporting their finding of impaired diastolic twisting mechanics - while Tan et $\mathrm{al}^{21}$ reported no change in the amount of untwisting occurring at any point during diastole. The percentage of LV untwisting occurring prior to mitral valve opening is of great interest, because any LV untwisting that occurs after mitral valve opening does not contribute to LV pressure decay and is therefore less efficient. ${ }^{8}$ 


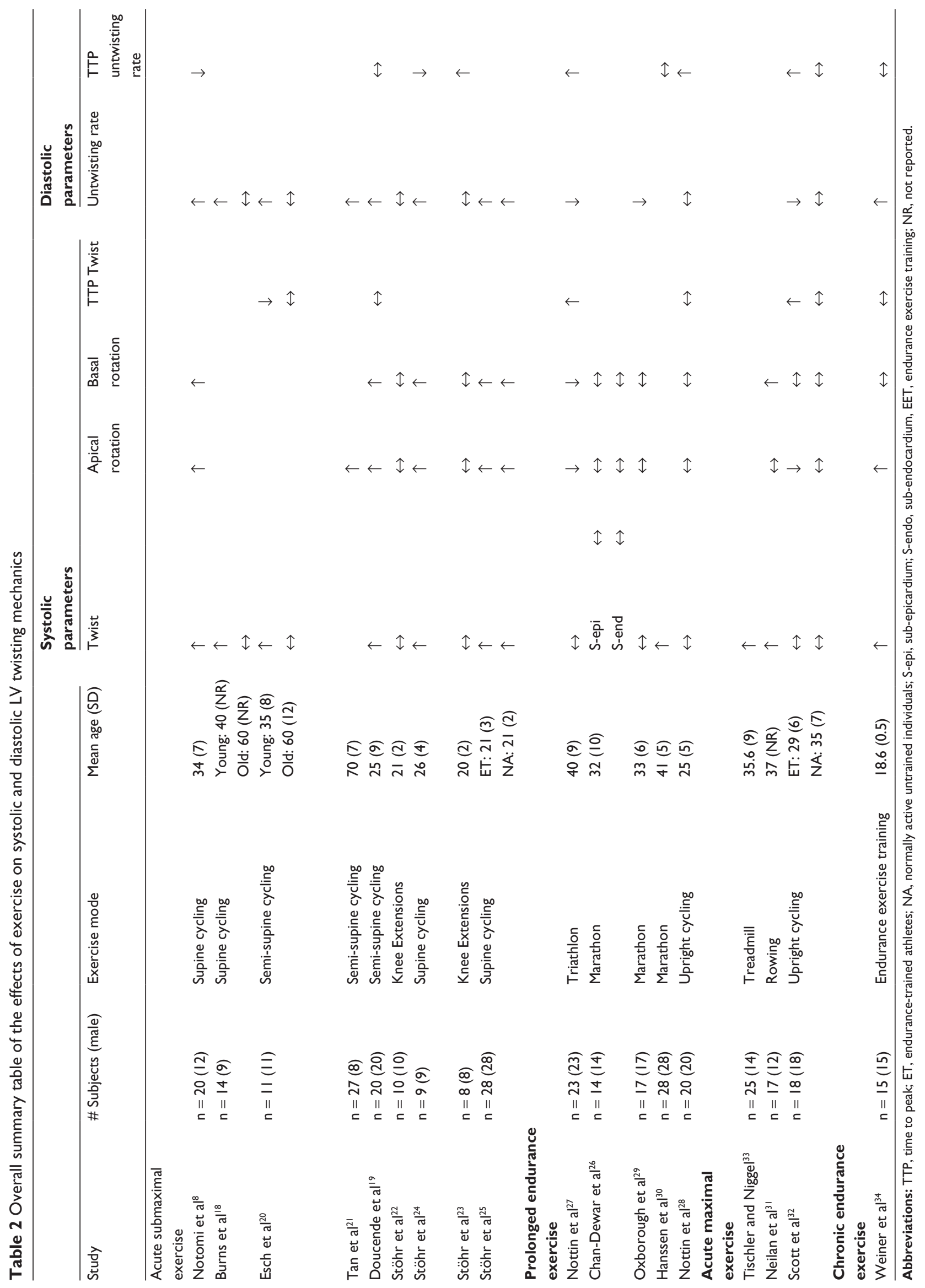




\section{Conclusion}

There is consistent support from level 4 and level 2 18,20,24,25,28 evidence that sub-maximal exercise results in enhanced systolic and diastolic LV twisting mechanics in individuals below age 40. The conflicting results present in the two level 4 studies likely arise from the use of low intensity exercise protocols. ${ }^{22,23}$ As eight investigations have examined the effects of sub-maximal exercise on LV twisting mechanics in younger individuals, the results can be interpreted to be relatively conclusive. However, the evidence in older individuals is less clear, and more work is needed examining LV twist during exercise across the human life span. Regardless, future studies should utilize comparable modes of exercise, and they should pay close attention to the clinical health of their participants. However, it is evident that the changes in systolic and diastolic LV twisting mechanics during submaximal exercise are influenced by age, as younger individuals are able to augment their LV function during exercise, while older individuals are not.

\section{Prolonged endurance exercise}

The effects of prolonged endurance exercise on resting LV systolic and diastolic twisting mechanics were investigated by five studies (102 total participants; approximate mean ages: $30-40$ years). The exercise protocols included three marathon races (involving $42.2 \mathrm{~km}$ of running), ${ }^{26,29,30}$ one ultra-long triathlon race (involving $3.8 \mathrm{~km}$ of swimming, $186 \mathrm{~km}$ of cycling, and $42.2 \mathrm{~km}$ of running), ${ }^{27}$ and one 120 -minute continuous cycling protocol. ${ }^{28}$ All investigations examined the changes in LV twisting mechanics pre- and post-exercise, with one investigation specifically examining the changes in the subendocardial and subepicardial layers of the myocardium. ${ }^{26}$

\section{Systolic parameters}

Prolonged endurance exercise primarily resulted in impaired LV systolic twisting mechanics. This was evident in the form of decreased peak systolic apical and basal rotations, ${ }^{27}$ increased time to peak apical and basal rotation, ${ }^{27}$ increased time to peak apical and basal rotation rates, ${ }^{28}$ a slight decrease in peak LV twist $(P=0.09),{ }^{27}$ delayed time to peak LV twist, ${ }^{26}$ and a decreased amount of LV twist occurring at the time of aortic valve closure. ${ }^{27,28}$ In contrast, however, one investigation reported augmented systolic twisting mechanics (increases in peak LV twist and twisting rate).$^{30}$ As preload has been previously demonstrated to influence systolic twisting mechanics, ${ }^{35}$ this disagreement could be the result of changes in preload before and after exercise. Accordingly, the two investigations that reported impaired systolic twisting mechanics also reported decreases in preload, ${ }^{27,29}$ while the investigation that reported augmented systolic twisting mechanics reported no change in preload. ${ }^{30}$ Finally, prolonged endurance exercise was also found to largely exert no effect on systolic twisting mechanics in the subendocardial and subepicardial layers of the myocardium, apart from an increase in peak subendocardial apical rotation rate. ${ }^{26}$

\section{Diastolic parameters}

Prolonged endurance exercise resulted primarily in impaired diastolic twisting mechanics. This was evident through a decrease in peak untwisting rate, ${ }^{27-29}$ an increase in time to peak untwisting rate, ${ }^{27-29}$ a decrease in peak diastolic apical and basal rotation rates, ${ }^{29}$ and a decrease in the amount of LV untwisting that occurred during isovolumic relaxation (indicating less efficient diastolic filling) ${ }^{29}$ Interestingly, the diastolic impairments observed after 120 minutes of endurance exercise ${ }^{28}$ were less severe than those observed after 850 minutes, ${ }^{28}$ suggesting a potential dose-response relationship between exercise duration and diastolic impairment. In addition, LV twist ${ }^{29}$ and systolic apical rotation ${ }^{30}$ were reported to remain elevated during early diastole, indicating a reduction in LV relaxation time and therefore a reduced time for diastolic untwisting and subsequent filling. These findings could also be interpreted as an increased time to peak LV twist, therefore suggesting an impairment in systolic twisting mechanics. Furthermore, Hanssen et $\mathrm{al}^{30}$ reported no evidence of impaired diastolic twisting function yet found a global decrease in diastolic filling. This finding, coupled with the elevated systolic apical rotation during early diastole, suggests that diastolic impairment may not always manifest in altered diastolic twisting mechanics. Finally, prolonged endurance exercise resulted in no changes in the twisting mechanics of the subendocardium and subepicardium. ${ }^{26}$

\section{Conclusion}

Level 4 evidence (indicating available evidence but without comparable groups) exists indicating that prolonged endurance exercise induces impaired systolic and diastolic LV twisting mechanics. ${ }^{27-29}$ Also, conflicting level 4 evidence exists indicating that prolonged endurance exercise results in augmented systolic twisting mechanics and unaltered diastolic twisting mechanics. ${ }^{30}$ However, this investigation also reported elevated measures of systolic twisting mechanics during diastole, suggesting a form of diastolic impairment consistent with previous reports. Finally, Level 4 evidence demonstrates that prolonged endurance exercise largely exerts no change in either systolic or diastolic twisting mechanics in the subendocardial and subepicardial layers 
of the myocardium. As there have only been five studies examining the changes in twisting mechanics after prolonged endurance exercise, the need for further research is clear. However, the relative consistency in the observed results tends to support the observed trends in the data. Of particular interest is the apparent effect of changes in preload on LV systolic twisting mechanics, a finding potentially resulting from excessive fluid loss or exercise-induced hypotension, which can occur during exercise of this duration and intensity.

\section{Acute maximal exercise}

Three articles investigated the effects of acute maximal exercise on resting LV twisting mechanics (60 total participants; approximate mean ages: $30-40$ years). All investigations measured LV function before and after exercise and each utilized a different exercise intervention, yet all reported achieving a maximal effort from their respective participants.

\section{Systolic parameters}

In response to maximal exercise, LV systolic twisting mechanics were found to increase, in the form of increases in peak LV twist, ${ }^{31,33}$ peak basal rotation, ${ }^{31}$ and slightly increased peak apical rotation $(P=0.07) .{ }^{31}$ Interestingly, one investigation that examined repeated bouts of maximal intensity exercise showed differing results. After performing 14 1-minute bouts at maximal exercise (separated by 2 minutes of recovery), no change in LV systolic twisting mechanics occurred in their untrained group, while the endurance-trained group experienced decreases in peak apical rotation rate and a delay in the time to peak twist. ${ }^{32}$ This disagreement is potentially due to the increased duration and intensity of the exercise protocol in comparison with the protocols involving single bouts at maximal intensity, and it draws similarities to the apparent doseresponse relationship between twisting mechanics and exercise duration found during prolonged endurance exercise.

\section{Diastolic parameters}

Diastolic LV twisting mechanics were only examined in one investigation, which reported evidence of reduced peak untwisting rate and an increased time to peak untwisting rate in endurance-trained individuals but not in untrained participants. ${ }^{32}$ This investigation also reported a reduced time interval between peak diastolic untwisting and LV filling (measured as peak circumferential strain rate) in endurance-trained individuals. As noted by the authors, peak diastolic untwist and LV filling are distinctly separated under normal resting conditions, as myocardial relaxation occurs before ventricular filling, ${ }^{36}$ and a reduction in this time interval therefore indicates impaired diastolic function. Interestingly, additional evidence exists that maximal exercise can also result in an attenuation of more traditional markers of LV diastolic function in endurance-trained participants. Neilan et $\mathrm{al}^{31}$ showed that there is a reversal in diastolic filling pattern in these individuals, perhaps suggesting that highly trained athletes are more susceptible to diastolic impairment following maximal exercise.

\section{Conclusion}

There is level 4 evidence that acute maximal exercise can result in augmented LV systolic twisting mechanics. ${ }^{30,32}$ Further to this, there is level 2 evidence that this same exercise type, albeit a more intense exercise protocol, can impair both systolic and diastolic twisting mechanics in endurance-trained individuals while exerting no changes in untrained participants. ${ }^{32}$ As only three studies exist examining the effects of acute maximal exercise on LV twisting mechanics, and as each investigation utilized a different exercise protocol and varying subject populations, more research is necessary that incorporates standardized exercise protocols and consistent population samples. However, despite these inconsistencies, it appears that endurance-trained individuals may experience a reduction in both systolic and diastolic LV twisting mechanics after maximal exercise while untrained individuals do not.

\section{Chronic endurance exercise}

One study investigated the effects of chronic endurance exercise on resting LV systolic and diastolic twisting mechanics in elite athletes ( 15 total participants; mean age: 19 years), ${ }^{34}$ utilizing 90 days of endurance exercise training (approximately 13.6 hours per week) as their exercise protocol. Measures of LV twisting mechanics were taken at rest before and after the training program.

\section{Systolic parameters}

Systolic LV twisting mechanics were found to be augmented following chronic endurance exercise in the form of increases in peak LV twist and peak systolic apical rotation. ${ }^{34}$

\section{Diastolic parameters}

Chronic endurance exercise resulted in enhanced LV diastolic twisting mechanics in the form of increased peak untwisting rate, increased peak diastolic apical rotation rate, and an increase in the percentage of untwisting occurring during isovolumic relaxation. ${ }^{34}$ This increase in early diastolic untwisting is likely the result of the increased LV systolic twist, which would generate greater elastic potential energy for release during early diastole. 


\section{Conclusion}

There is level 4 evidence that chronic endurance exercise results in augmented LV systolic and diastolic twisting mechanics. ${ }^{34}$ Although this is the only study of its kind, previous investigations have examined resting LV systolic and diastolic twisting mechanics in elite athletes and untrained individuals, reporting reduced peak LV twist $\mathrm{t}^{37,38}$ and both reduced $^{38}$ and unchanged ${ }^{37} \mathrm{LV}$ diastolic untwisting in elite athletes. This disagreement, recognized by Weiner et al, ${ }^{34}$ was attributed to differences in study populations and study designs, as Nottin et $a l^{37}$ and Zócalo et $a l^{38}$ examined slightly older individuals and used a cross-sectional study design that could have collected data from the athletes while they were in a period of detraining.

\section{Discussion}

Significant contention exists regarding changes in LV twisting mechanics occurring in response to exercise. This debate has resulted in several publications (see References section for examples). ${ }^{111,12,39,44}$ The purpose of this review was to systematically summarize the existing published evidence examining the influence of exercise on LV twisting mechanics. Separation of the articles according to exercise type was essential in order to adequately compare investigations, and this yielded mixed results, with no category having complete agreement. However, despite these observed inconsistencies, some intriguing trends were found in the literature.

First, sub-maximal exercise appears to result in augmented LV systolic and diastolic twisting mechanics in only younger individuals (below age 40). Given that exercise produces increases in heart rate and contractility, both previously demonstrated to increase LV twisting mechanics, ${ }^{40,41}$ this finding can be expected. However, the increases in venous return (preload) and systolic blood pressure (often used as an indicator of afterload) that also occur with exercise, and have been demonstrated to influence LV twisting mechanics through opposing mechanisms, ${ }^{42,43}$ could account for some of the observed result variability. With regards to the effects of age on LV twisting mechanics during exercise previous research has demonstrated the influence of age on resting LV twisting mechanics, ${ }^{45,46}$ yet the inability of older individuals to enhance LV twisting mechanics has not been fully explained. One theory attributes this age-related change to a decreased 'twisting reserve' in older individuals due to higher resting LV twist, therefore reducing the capacity of the LV to further twist/ untwist during exercise, ${ }^{18}$ yet more research is needed to thoroughly investigate this theory.
Second, prolonged endurance exercise appears to impair both LV systolic and diastolic twisting mechanics. LV function during systole and diastole has been well documented to decrease following endurance exercise through traditional measures such as ejection fraction and $\mathrm{E} / \mathrm{A}$ ratio (the ratio between the early maximal ventricular filling velocity and the late filling velocity), ${ }^{12,47}$ thereby providing support for this observed decrease in twisting mechanics. Interestingly, there also appears to be a dose-response relationship between the duration and intensity of endurance exercise and the level of twisting impairment. Again, this is supported by previous research using traditional measures of LV function that demonstrate that increases in exercise duration and intensity are proportional to the level of cardiac function impairment. ${ }^{48}$ Changes in cardiac loading after endurance exercise were also found to potentially exert an effect on systolic twisting mechanics, supporting the idea that LV twisting mechanics are indeed load-dependent, manifested in these studies as changes in end diastolic volume.

Finally, a general trend found throughout all exercise types is the linkage between the systolic and diastolic components of LV twisting and untwisting. In other words, if systolic twisting was impaired (or improved), it resulted in impaired (or improved) diastolic untwisting. This finding suggests that the systolic-diastolic rotational coupling of the left ventricle, through the production and release of elastic potential energy, ${ }^{8}$ holds true for the various exercise modalities. In fact, systolic-diastolic LV rotational coupling has also been widely reported in various heart diseases. ${ }^{1}$ As it appears that systolic function cannot be altered without similar implications on diastolic function, or vise versa, the connectedness between systole and diastole through LV twisting mechanics provides an important avenue for future cardiac research. ${ }^{49}$

\section{Methodological considerations}

The presence of conflicting evidence in many of the exercise categories is potentially explained by some important methodological considerations. The methodological quality of the analyzed investigations was low to moderate and the evidence was primarily rated at level 4. Methodological quality could be improved in future studies through the use of control groups (absent in 15 of the 18 studies), and their absence in the current body of literature is difficult to explain - perhaps because of unreported challenges such as an increased amount of time required to analyze echocardiographic images, or difficulty with participant recruitment. In addition, there was significant variability in the exercise protocols and subject characteristics present within each exercise 
type. The inconsistent durations and intensities of the exercise protocols across studies of the same exercise type are likely to account for some of the observed conflicting results. As increases in exercise duration and intensity have already been demonstrated to directly relate to changes in cardiac function during endurance exercise, ${ }^{48}$ it is conceivable that variations in the duration and intensity of the exercise protocols used in the other exercise types could exert similar effects. The use of different exercise protocols also creates different conditions of cardiac loading. In the submaximal exercise category, for example, participants exercised in the supine, semi-supine, and upright postural positions - each resulting in differing levels of venous return and therefore preload. Given the aforementioned influence of changes in cardiac loading on LV twisting mechanics, the use of inconsistent exercise protocols is an important methodological consideration. Furthermore, previous research has demonstrated that the LV twisting mechanics of endurance-trained athletes and untrained individuals are affected differently by reductions in preload resulting from an orthostatic challenge. ${ }^{50}$ This finding highlights the importance of not only cardiac loading but also participant training status, as variations in the level of participant fitness between studies may further contribute to the disagreement. Another potential confounding factor and methodological consideration is the use of sample populations consisting of mixed sexes, as females are known to exhibit altered cardiac responses to both acute ${ }^{51}$ and prolonged ${ }^{52}$ exercise in comparison with males. Finally, variations in transducer probe position during the collection of apical short-axis echocardiographic images have been demonstrated to significantly alter the calculation of LV apical rotation. ${ }^{53}$ The use of trained personnel to collect and analyze the data in order to reduce measurement error and result variability is therefore essential.

\section{Conclusion}

The measurement of LV twisting mechanics during exercise is becoming increasingly prevalent and has resulted in considerable debate. The study of the rotational properties of the left ventricle provides important insight into cardiac function during exercise that traditional measures of LV function cannot. Despite some important methodological considerations present in the available literature, there are significant trends in each exercise category that should be considered. Most important, the rotational coupling of the left ventricle between systole and diastole holds true in all exercise types, as LV systolic and diastolic twisting mechanics were impaired (or improved) concomitantly. Future research should include standardized exercise protocols and consistent subject characteristics in order to limit the methodologicalinduced variability in the observed results.

\section{Disclosure}

The authors report no conflicts of interest in this work.

\section{References}

1. Esch BT, Warburton DER. Left ventricular torsion and recoil: implications for exercise performance and cardiovascular disease. J Appl Physiol. 2009;106(2):362-369.

2. Streeter DD, Spotnitz HM, Patel DP, Ross J, Sonnenblick EH. Fiber orientation in the canine left ventricle during diastole and systole. Circ Res. 1969;24:339-347.

3. Beyar R, Sideman S. Effect of the twisting motion on the nonuniformities of transmyocardial fiber mechanics and energy demand: a theoretical study. IEEE Trans Biomed Eng. 1985;32(10):764-769.

4. Nikolic SD, Yellin EL, Dahm M, Pajaro O, Frater RW. Relationship between diastolic shape (eccentricity) and passive elastic properties in canine left ventricle. Am J Physiol. 1990;259(2 Pt 2):H457-H463.

5. Fukuda N, Sasaki D, Ishiwata S, Kurihara S. Length dependence of tension generation in rat skinned cardiac muscle: role of titin in the Frank-Starling mechanism of the heart. Circulation. 2001;104(14): $1639-1645$.

6. Granzier H, Wu Y, Siegfried L, LeWinter M. Titin: physiological function and role in cardiomyopathy and failure. Heart Fail Rev. 2005;10(3):211-223.

7. Rademakers FE, Buchalter MB, Rogers WJ, et al. Dissociation between left ventricular untwisting and filling: accentuation by catecholamines. Circulation. 1992;85(4):1572-1581.

8. Notomi Y, Martin-Miklovic MG, Oryszak SJ, et al. Enhanced ventricular untwisting during exercise: a mechanistic manifestation of elastic recoil described by Doppler tissue imaging. Circulation. 2006;113(21):2524-2533.

9. Helle-Valle T, Crosby J, Edvardsen T, et al. New noninvasive method for assessment of left ventricular rotation: speckle tracking echocardiography. Circulation. 2005;112(20):3149-3156.

10. Notomi Y, Lysyansky P, Setser RM, et al. Measurement of ventricular torsion by two-dimensional ultrasound speckle tracking imaging. $\mathrm{J} \mathrm{Am}$ Coll Cardiol. 2005;45(12):2034-2041.

11. George KP, Naylor LH, Whyte GP, Shave RE, Oxborough D, Green DJ. Diastolic function in healthy humans: non-invasive assessment and the impact of acute and chronic exercise. Eur J Appl Physiol. 2010;108(1):1-14.

12. Oxborough D, Birch K, Shave R, George K. "Exercise-induced cardiac fatigue": a review of the echocardiographic literature. Echocardiography. 2010;27(9):1130-1140.

13. Warburton DER, Charlesworth S, Ivey A, Nettlefold L, Bredin SS. A systematic review of the evidence for Canada's Physical Activity Guidelines for Adults. Int J Behav Nutr Phys Act. 2010;7:39.

14. Downs SH, Black N. The feasibility of creating a checklist for the assessment of the methodological quality both of randomised and nonrandomised studies of health care interventions. J Epidemiol Community Health. 1998;52(6):377-384.

15. Prince SA, Adamo KB, Hamel M, Hardt J, Gorber SC, Tremblay M. A comparison of direct versus self-report measures for assessing physical activity in adults: a systematic review. Int J Behav Nutr Phys Act. 2008;5:56.

16. Eng JJ, Teasell RW, Miller WC, et al. Spinal Cord Injury Rehabilitation Evidence: method of the SCIRE Systematic Review. Top Spinal Cord Inj Rehabil. 2007;13(1):1-10.

17. Sackett DL, Straus SE, Richardson WS, Rosenberg W, Haynes RB Evidence-Based Medicine: How to Practice and Teach EBM, 2nd ed. New York (NY): Churchill Livingstone; 2000. 
18. Burns AT, La Gerche A, MacIsaac AI, Prior DL. Augmentation of left ventricular torsion with exercise is attenuated with age. J Am Soc Echocardiogr. 2008;21(4):315-320.

19. Doucende G, Schuster I, Rupp T, et al. Kinetics of left ventricular strains and torsion during incremental exercise in healthy subjects: the key role of torsional mechanics for systolic-diastolic coupling. Circ Cardiovasc Imaging. 2010;3(5):586-594.

20. Esch BT, Scott JM, Warburton DER, et al. Left ventricular torsion and untwisting during exercise in heart transplant recipients. J Physiol. 2009;587(Pt 10):2375-2386.

21. Tan YT, Wenzelburger F, Lee E, et al. The pathophysiology of heart failure with normal ejection fraction: exercise echocardiography reveals complex abnormalities of both systolic and diastolic ventricular function involving torsion, untwist, and longitudinal motion. J Am Coll Cardiol. 2009;54(1):36-46.

22. Stöhr EJ, González-Alonso J, Pearson J, et al. Effects of graded heat stress on global left ventricular function and twist mechanics at rest and during exercise in healthy humans. Exp Physiol. 2011;96(2): 114-124.

23. Stöhr EJ, González-Alonso J, Pearson J, et al. Dehydration reduces left ventricular filling at rest and during exercise independent of twist mechanics. J Appl Physiol. 2011;111(3):891-897.

24. Stöhr EJ, González-Alonso J, Shave R. Left ventricular mechanical limitations to stroke volume in healthy humans during incremental exercise. Am J Physiol Heart Circ Physiol. 2011;301(2):H478-H487.

25. Stöhr EJ, McDonnell B, Thompson J, et al. Left ventricular mechanics in humans with high aerobic fitness: adaptation independent of structural remodelling, arterial haemodynamics and heart rate. J Physiol. 2012;590(Pt 9):2107-2119.

26. Chan-Dewar F, Oxborough D, Shave R, Gregson W, Whyte G, George K. Left ventricular myocardial strain and strain rates in sub-endocardial and sub-epicardial layers before and after a marathon. Eur J Appl Physiol. 2010;109(6):1191-1196.

27. Nottin S, Doucende G, Schuster I, Tanguy S, Dauzat M, Obert P. Alteration in left ventricular strains and torsional mechanics after ultralong duration exercise in athletes. Circ Cardiovasc Imaging. 2009;2(4):323-330.

28. Nottin S, Ménétrier A, Rupp T, Boussuges A, Tordi N. Role of left ventricular untwisting in diastolic dysfunction after long duration exercise. Eur J Appl Physiol. 2011;112(2):525-533.

29. Oxborough D, Whyte G, Wilson M, et al. A depression in left ventricular diastolic filling following prolonged strenuous exercise is associated with changes in left atrial mechanics. J Am Soc Echocardiogr. 2010;23(9):968-976.

30. Hanssen H, Keithahn A, Hertel G, et al. Magnetic resonance imaging of myocardial injury and ventricular torsion after marathon running. Clin Sci (Lond). 2011;120(4):143-152.

31. Neilan TG, Ton-Nu TT, Jassal DS, et al. Myocardial adaptation to short-term high-intensity exercise in highly trained athletes. J Am Soc Echocardiogr. 2006;19(10):1280-1285.

32. Scott JM, Esch BT, Haykowsky MJ, et al. Effects of high intensity exercise on biventricular function assessed by cardiac magnetic resonance imaging in endurance trained and normally active individuals. $\mathrm{Am} \mathrm{J}$ Cardiol. 2010;106(2):278-283.

33. Tischler M, Niggel J. Left ventricular systolic torsion and exercise in normal hearts. J Am Soc Echocardiogr. 2003;16(6):670-674.

34. Weiner RB, Hutter AM Jr, Wang F, et al. The impact of endurance exercise training on left ventricular torsion. JACC Cardiovasc Imaging. 2010;3(10):1001-1009.

35. Gibbons Kroeker CA, Tyberg JV, Beyar R. Effects of load manipulations, heart rate, and contractility on left ventricular apical rotation: an experimental study in anesthetized dogs. Circulation. 1995;92(1):130-141.
36. Nagel E, Stuber M, Burkhard B, et al. Cardiac rotation and relaxation in patients with aortic valve stenosis. Eur Heart J. 2000;21(7):582-589.

37. Nottin S, Doucende G, Schuster-Beck I, Dauzat M, Obert P. Alteration in left ventricular normal and shear strains evaluated by 2D-strain echocardiography in the athlete's heart. J Physiol. 2008;586(Pt 19): 4721-4733.

38. Zócalo Y, Bia D, Armentano RL, et al. Assessment of training-dependent changes in the left ventricle torsion dynamics of professional soccer players using speckle-tracking echocardiography. Conf Proc IEEE Eng Med Biol Soc. 2007;2007:2709-2712.

39. Weiner RB, Baggish AL. Left ventricular torsion: feeling the heat. Exp Physiol. 2011;96(2):71-72.

40. Hansen DE, Daughters GT, Alderman EL, Ingels NB, Stinson EB, Miller DC. Effect of volume loading, pressure loading, and inotropic stimulation on left ventricular torsion in humans. Circulation. 1991; 83(4):1315-1326.

41. Moon MR, Ingels NB, Daughters GT, Stinson EB, Hansen DE, Miller DC. Alterations in left ventricular twist mechanics with inotropic stimulation and volume loading in human subjects. Circulation. 1994;89(1):142-150.

42. Dong SJ, Hees PS, Huang WM, Buffer SA, Weiss JL, Shapiro EP. Independent effects of preload, afterload, and contractility on left ventricular torsion. Am J Physiol. 1999;277(3 Pt 2):H1053-H1060.

43. MacGowan GA, Burkhoff D, Rogers WJ, et al. Effects of afterload on regional left ventricular torsion. Cardiovasc Res. 1996;31(6): 917-925.

44. Phillips AA, Warburton DE. Letter to the editor: "Left ventricular mechanical limitations to stroke volume in healthy humans during incremental exercise". Am J Physiol Heart Circ Physiol. 2012;302(1):H375; author reply $\mathrm{H} 376-\mathrm{H} 377$.

45. Notomi Y, Srinath G, Shiota T, et al. Maturational and adaptive modulation of left ventricular torsional biomechanics: Doppler tissue imaging observation from infancy to adulthood. Circulation. 2006;113(21):2534-2541.

46. Nakai H, Takeuchi M, Nishikage T, Kokumai M, Otani S, Lang RM. Effect of aging on twist-displacement loop by 2-dimensional speckle tracking imaging. J Am Soc Echocardiogr. 2006;19(7):880-885.

47. Shave R, George K, Whyte G, Hart E, Middleton N. Postexercise changes in left ventricular function: the evidence so far. Med Sci Sports Exerc. 2008;40(8):1393-1399.

48. Middleton N, Shave R, George K, Whyte G, Hart E, Atkinson G. Left ventricular function immediately following prolonged exercise: a metaanalysis. Med Sci Sports Exerc. 2006;38(4):681-687.

49. Pasipoularides A. LV twisting and untwisting in HCM: ejection begets filling; diastolic functional aspects of HCM. Am Heart J. 2011;162(5):798-810.

50. Esch BT, Scott JM, Haykowsky MJ, et al. Changes in ventricular twist and untwisting with orthostatic stress: endurance athletes versus normally active individuals. J Appl Physiol. 2010;108(5):1259-1266.

51. Davis SN, Galassetti P, Wasserman DH, Tate D. Effects of gender on neuroendocrine and metabolic counterregulatory responses to exercise in normal man. J Clin Endocrinol Metab. 2000;85(1):224-230.

52. Scott JM, Esch BTA, Haykowsky MJ, et al. Sex differences in left ventricular function and beta-receptor responsiveness following prolonged strenuous exercise. J Appl Physiol. 2007;102(2):681-687.

53. Van Dalen BM, Vletter WB, Soliman OI, ten Cate FJ, Geleijnse ML. Importance of transducer position in the assessment of apical rotation by speckle tracking echocardiography. J Am Soc Echocardiogr. 2008;21(8):895-898. 


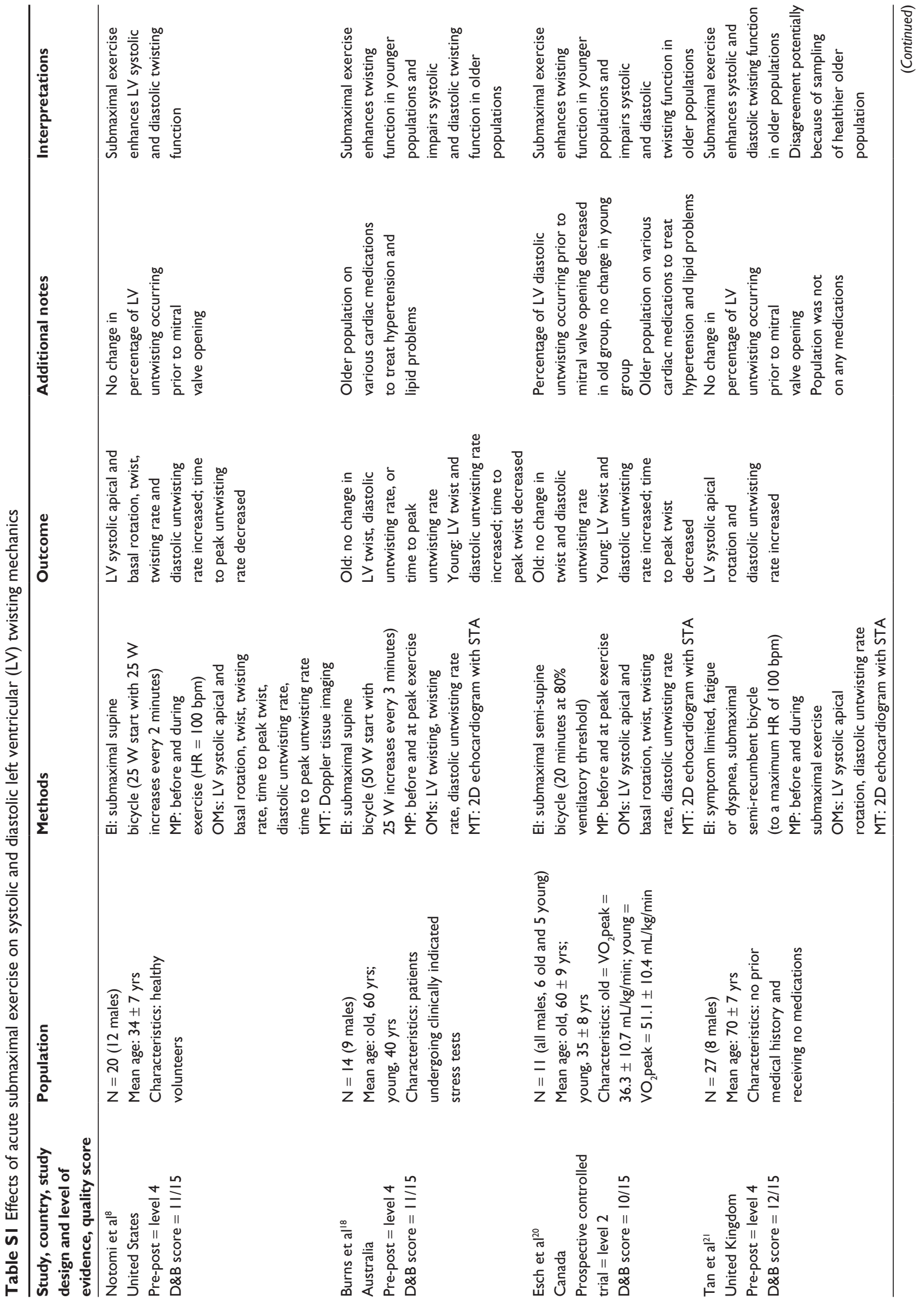




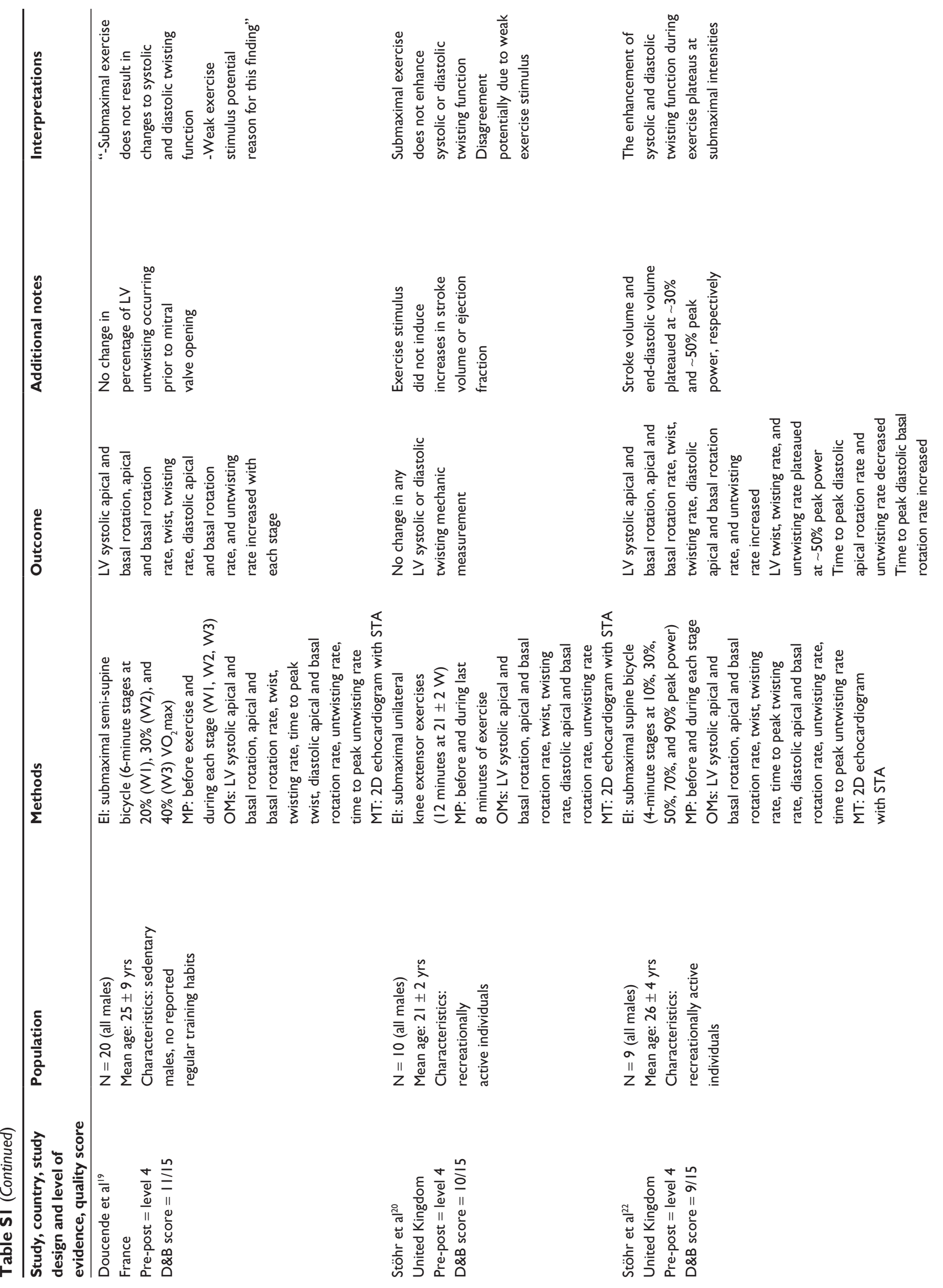



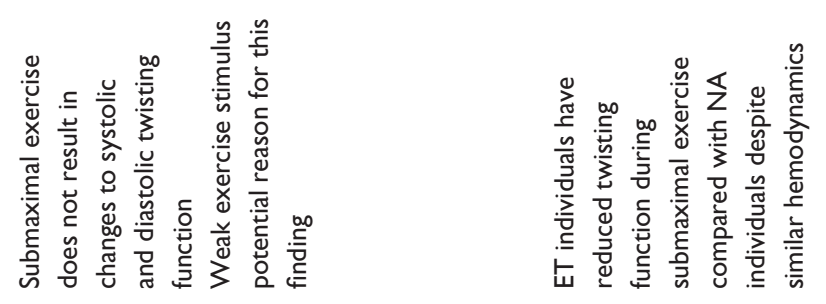

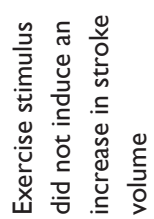
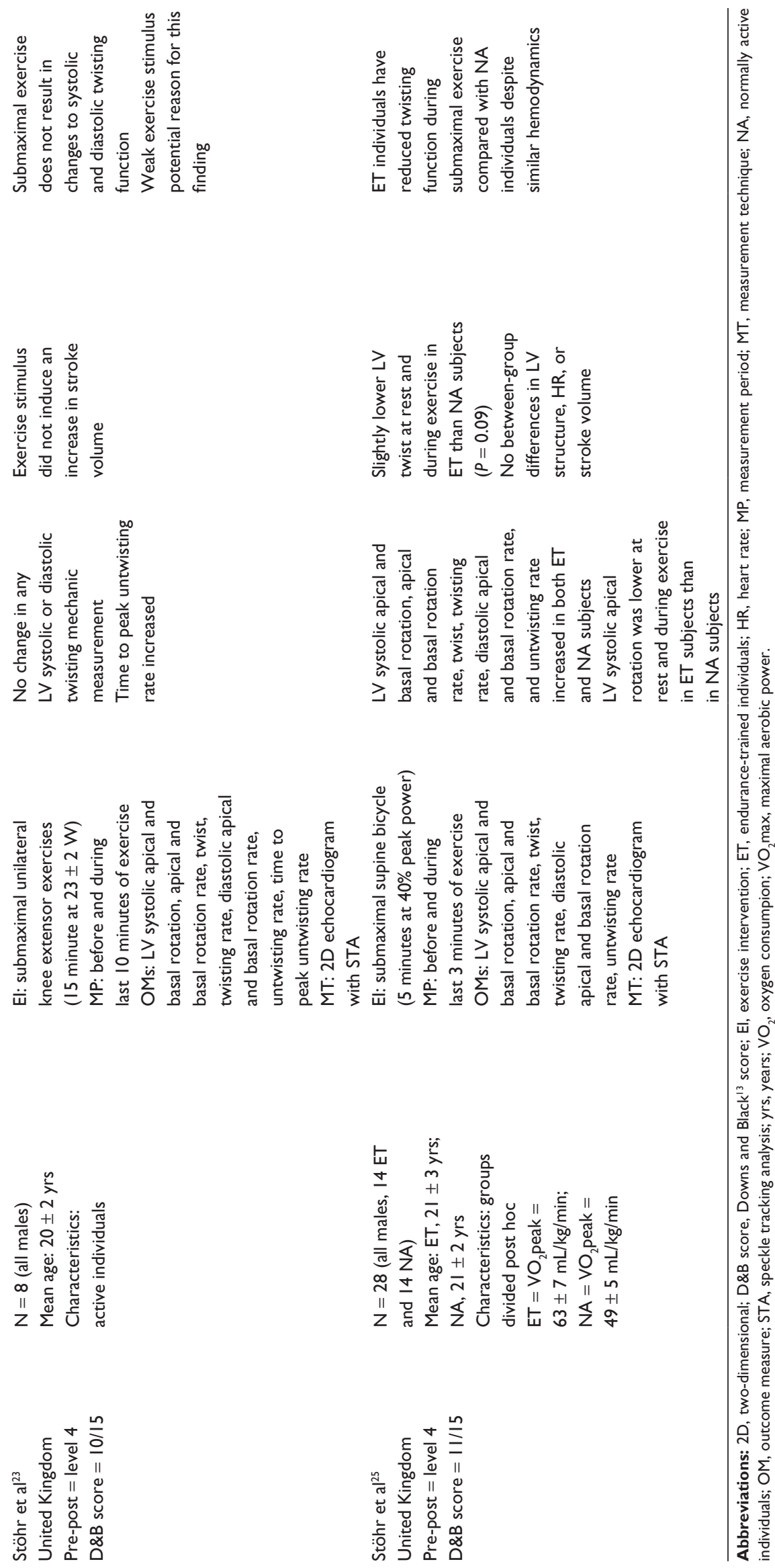


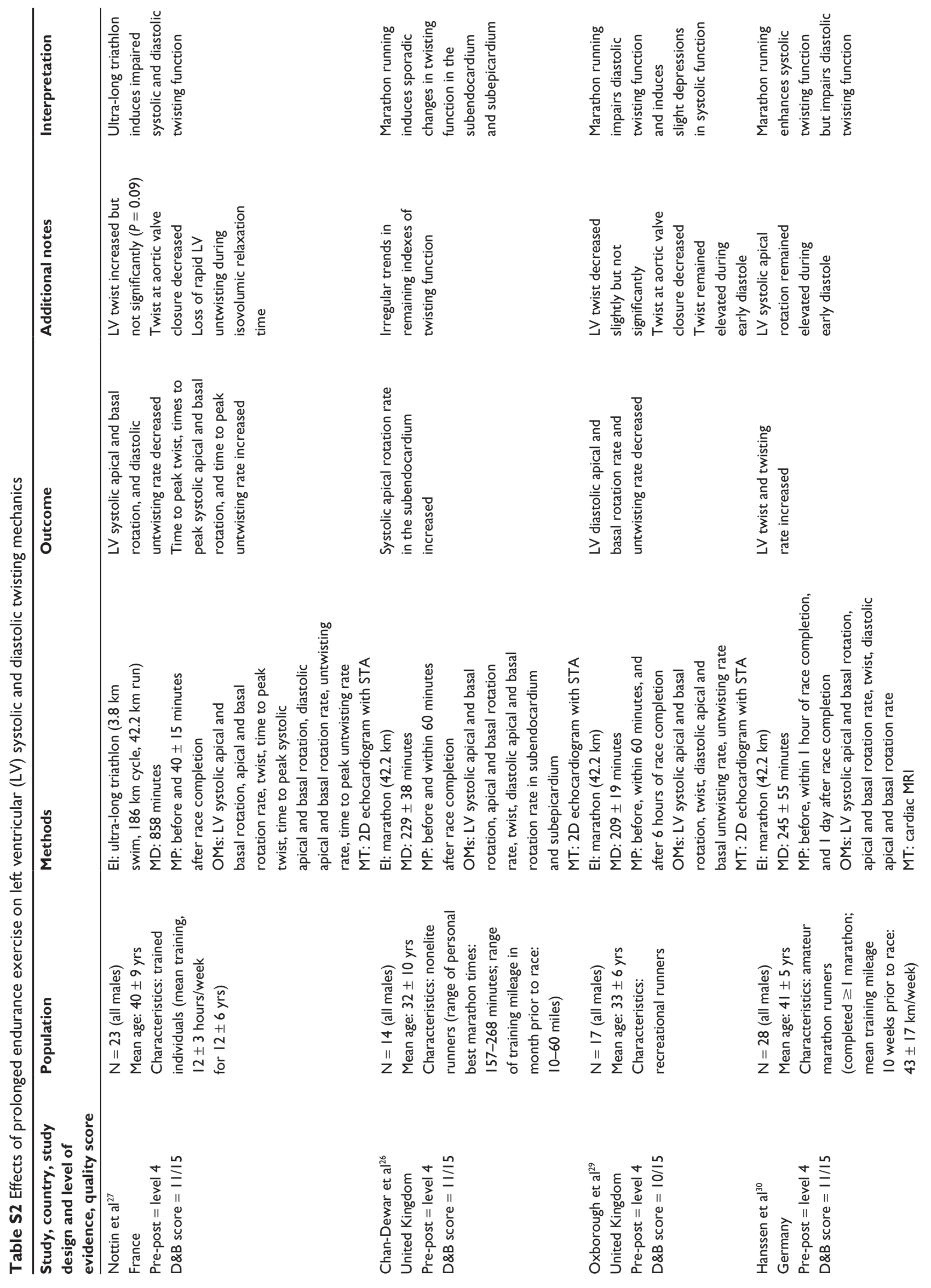



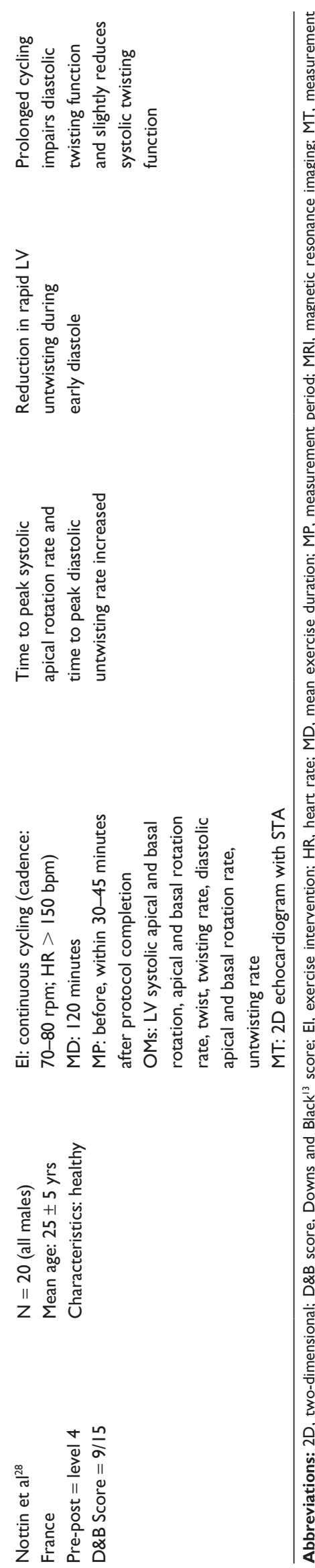


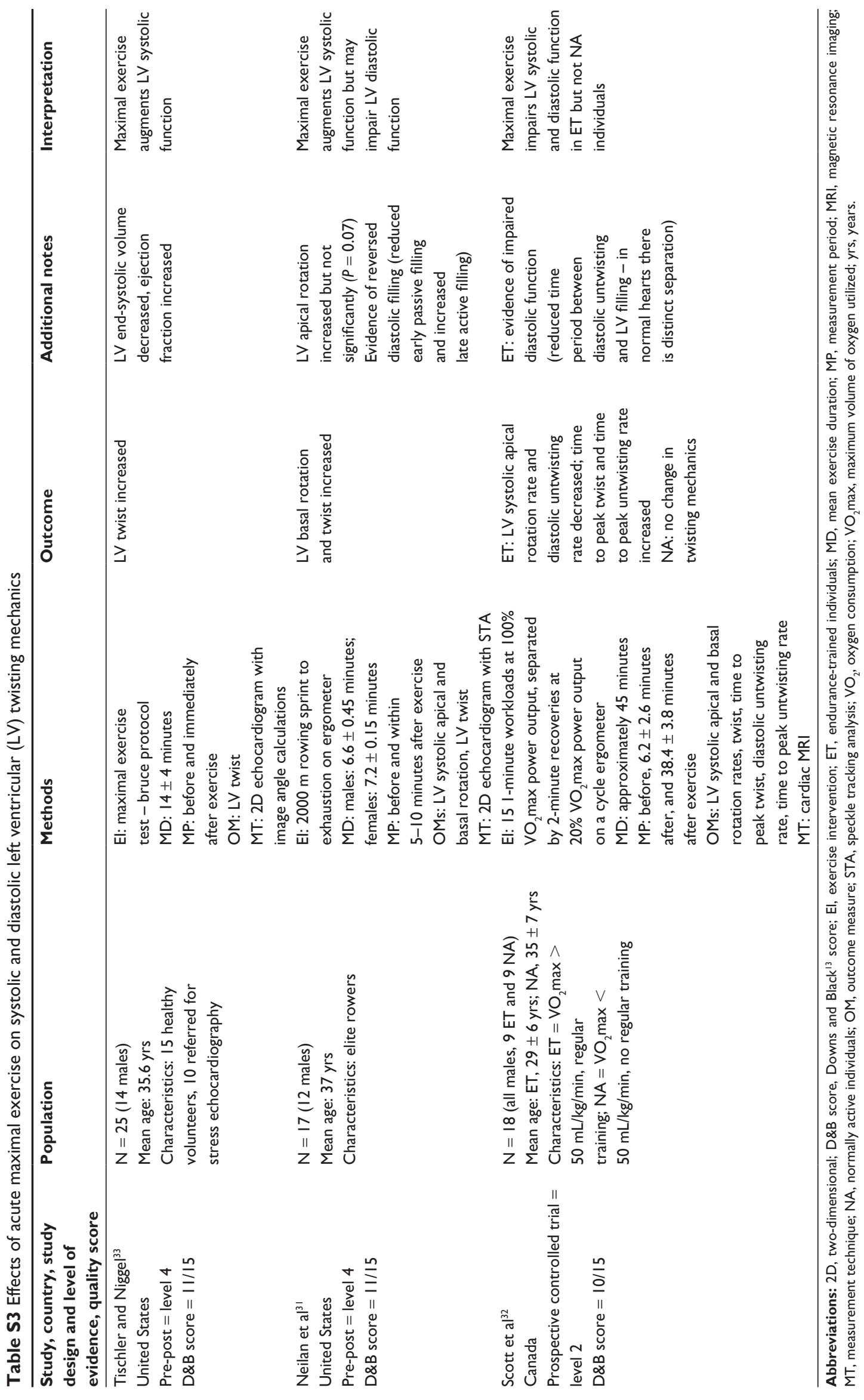




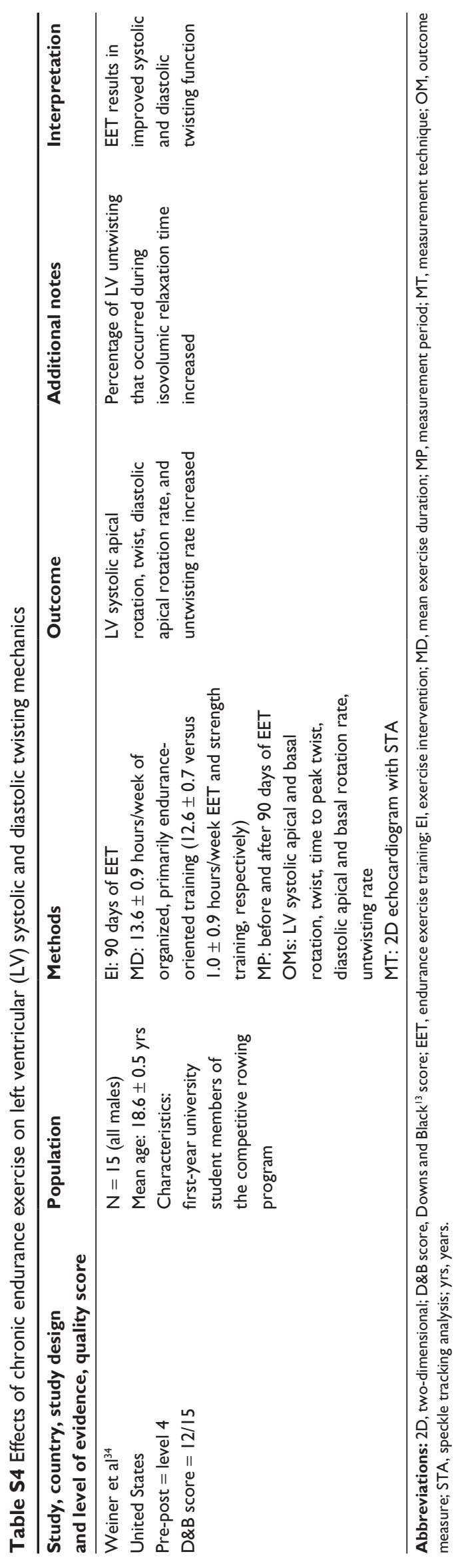




\section{Publish your work in this journal}

Open Access Journal of Sports Medicine is an international, Visit http://www.dovepress.com/testimonials.php to read real quotes peer-reviewed, open access journal publishing original research, from published authors.

reports, reviews and commentaries on all areas of sports

medicine. The manuscript management system is completely

online and includes a very quick and fair peer-review system.

Submit your manuscript here: http://www.dovepress.com/open-access-journal-of-sports-medicine-journal 